\title{
The SLC as a Second Generation Linear Collider*
}

\author{
J.E. Spencer \\ Stanford Linear Accelerator Center, Stanford University, Stanford, CA 94309 USA
}

\section{Abstract}

With enough luminosity, the SLC could contribute to most of the high energy physics of current interest such as new hadrons, quark molecules, glueballs and studies of the Standard Model and Minimal Supersymmetric SM in the form of particle searches for the lowest mass Higgs or selectron or tests of the point-like predictions for the $W, Z$ or $\tilde{e}_{R}$. Some experiments require alternative incident channels such as $e \gamma$ and $\gamma \gamma$ but only modest increases in energy. Just as the SLC was a prototype for the NLC, it could also be a prototype for a general (or gamma) linear collider - a GLC. Because the main problem is luminosity, we give a scaling relation based on multiple bunches per RF pulse. We then ask what is possible for the SLC in terms of bunch and train current, emittance and energy at the IP. The results suggest a phased development with the Higgs as a possible last step requiring a luminosity $\mathcal{L} \geq 10^{32}$.

\section{Introduction}

The assumption is made that before an NLC is approved, a working prototype is necessary that can demonstrate the feasibility of a general purpose linear collider capable of $\vec{e}^{ \pm} \vec{e}^{ \pm}, \vec{\gamma} \vec{e}^{ \pm}$and $\vec{\gamma} \vec{\gamma}$ incident channels. It is also argued that new physics is available over a range of energies starting at a few $\mathrm{GeV}$ by modifying the SLC to provide such channels and that this physics is hard to achieve elsewhere [1].

We begin with a discussion of the SLC relative to the next generation of linear colliders. Because the $e^{+} e^{-}$option has been verified, we concentrate on the other channels since positrons are an unnecessary complication for a single linac. While we don't preclude that option, our primary goal is to optimize the $\vec{e}-\vec{e}$-luminosity $\left(\equiv \mathcal{L}_{e e}\right.$ ) because this channel is used to produce the others [1].

From the expression for $\mathcal{L}$, one wants to maximize the available charge in a single bunch $N_{B}$, then the number of bunches in a train $n_{B}$ and finally the RF rep-rate $f_{T}$ :

$$
\mathcal{L}=\frac{f_{T} n_{B} N_{B}^{2} H_{D}}{4 \pi \sigma_{x} \sigma_{y}} \zeta \rightarrow \frac{f_{T} n_{B} N_{B}^{2}}{4 \pi \epsilon_{n} \beta^{*}} \gamma \propto \frac{P_{b} N_{B}}{\epsilon_{n} \beta^{*}}
$$

where $P_{b}$ is beam power and $\epsilon_{n}$ the invariant emittance.

To get more high quality charge through the linac and arcs we consider multibunch operation and coalescence. The reduced energy from beam loading reduces emittance growth in the arcs and is ideal for initial experiments [1]. For subsequent phases, energy can be restored in various ways e.g. by adding 'afterburners' between the arcs and final focus. Other ways to increase the energy and/or the charge/bunch are discussed in terms of a luminosity budget that indicates $\mathcal{L} \geq 10^{32}$ is possible.

"Work supported by the Department of Energy contract DE-AC03-76SF00515

\section{Comparison to the Next Generation}

A major concern in the design of the next generation of linear colliders is the effect of transverse and longitudinal wakefields that depend on the characteristics of the bunch and accelerating structure. This arises from the higher beam energies and powers that are required and the fact that to achieve these one is forced to higher RF frequencies and accelerating gradients. Because wake effects depend on the number of apertures, inversely on their area and the amount and distribution of charge in a single bunch, they become increasingly important.

While one would like all the charge/RF pulse in one bunch, this is impractical-for incident emittance, energy spread as well as the beamstrahlung. However, the band(width of the control system and the stability of the accelerator and its various subsystems relates more to the RF rep-rate $f_{T}$. A practical solution would appear to be multibunch trains i.e. to partition the total charge/pulse into a more continuous flow. As a result, most current designs propose to accelerate several bunches per RF pulse with somewhat lower bunch charge $N_{B}$ than the SLC in order to improve energy efficiency and average luminosity. Any required luminosity then has to come from increasing $P_{b}$ i.e. $P_{R F}$ and $P_{A C}$ (or $n_{B}$ and $f_{T}$ ) [2].

Thus, because preceding bunches perturb the effective emittance, energy and spread of the following bunches, multibunch beam loading and phase compensation must be considered to determine the best distribution of charge over each RF pulse. The next question is whether the bunches can be coalesced after acceleration when the physical apertures are larger. This would combine the best aspects of the different designs and reduce the pressure to maximize $N_{B}$. Because it is difficult to maintain the emittance with conventional magnetic compression one can consider other methods analogous to FEL bunching structures, fast phase switching techniques [3] or transient wake effects [4].

\section{SLC Improvements/Limitations}

We now discuss these issues relative to the SLC. We also ask how to avoid the damping rings and take advantage of the ares for bunch compression/coelescence. One problem in using the arcs is emittance growth which is discussed.

We will assume that we are dealing solely with $e^{-} e^{-}$ beams since they provide interesting physics and are used to produce the photon channels. A major advantage of electrons is that they can be polarized so we can control. the distribution of energy and polarization in all incident channels [1]. Another advantage is that it allows us to avoid damping rings i.e. simplify multibunch operation if a low emittance RF gun for polarized electrons was available. 


\section{A. The Generalized Luminosity $\mathcal{L}$}

The luminosity given above was for round beams where $H_{D}$ was the pinch parameter and $\zeta$ was the efficiency of the collision in terms of crossing angles and other parameter conflicts. Since we didn't label this $\mathcal{L}_{e e}$ we could also include the efficiency of converting $e \rightarrow \gamma$. For multibunch operation, we introduce a crossing angle and design the FF quads accordingly. This decreases $\zeta$ so that we can then introduce variable, crab-crossing cavities or RF deflectors that rotate the beams to the appropriate orientation at the IP or CP (the eq conversion point).

The rms spot size $\sigma$ at a round focus is

$\sigma^{*}=\left(\frac{\epsilon_{n} \beta^{*}}{\gamma}\right)^{\frac{1}{2}}=\frac{1}{\sqrt{\epsilon_{1}(\mathrm{GeV})}} \mu \mathrm{m} \quad\left(\epsilon_{n}=2 \mu \mathrm{m}, \beta^{*}=1 \mathrm{~mm}\right)$.

With these characteristics in a 'second generation' collider we would then expect an electron luminosity

$$
\mathcal{L}_{e e} \approx \epsilon_{1}(\mathrm{GeV}) \cdot 10^{31} \mathrm{~cm}^{-2} \mathrm{~S}^{-1} \quad\left(f_{T}=120, f_{B}=100, N_{B}=10^{10}\right) .
$$

Thus, $\mathcal{L}$ varies linearly with incident beam energy if the other parameters are energy independent.

We argue that the product of the parameters $H$ and $\zeta$ may be made no worse than 0.2-1.0. Besides simplicity, this is why we consider round beams and ignore $H$ and $\zeta$ except for conversion efficiency. To get $\mathcal{L}_{e e} \geq 5 \cdot 10^{32}$ we need an incident e-beam energy $\epsilon_{1} \geq 50 \mathrm{GeV}$ - the nominal energy of the SLC.

We get $\mathcal{L}_{e \gamma}$ or $\mathcal{L}_{\gamma \gamma}$ from $\mathcal{L}_{e \ell}$ by folding it with the conversion efficiencies and a luminosity coefficient $\eta(z)$. If we can take the e-focus at $z=0$, then the luminosity coefficient at $z$ relates to the electron size $\sigma(z)$ at that point. Using

$$
\beta(z)=\beta^{*}+\frac{z^{2}}{\beta^{*}} \Rightarrow \sigma(z)=\sigma^{*} \frac{z}{\beta^{*}} \simeq(f / D) \lambda
$$

implies small conversion distances for efficient use of laser power or good conversion efficiency and luminosity i.e. $z$ $\approx\left(\beta^{*} / \sigma^{*}\right)(f / D) \lambda$. Increasing laser intensity increases the strong-field QED processes that we want to avoid.

In Compton collisions and other radiative processes, the lowest energy photons scatter at the largest angles i.e.

$$
\theta(\omega)=\theta_{\circ} \sqrt{\frac{\omega_{\max }}{\omega}-1} \quad\left(\theta_{\circ}=\sqrt{x+1} / \gamma_{1}\right)
$$

so that

$$
\eta(z) \equiv \frac{z \theta_{0}}{\sigma^{*}} \approx \frac{1}{2}\left(\frac{z}{\mathrm{~mm}}\right)\left(\frac{\mathrm{GeV}}{\epsilon_{\mathrm{i}}}\right)\left(\frac{\mu \mathrm{m}}{\sigma^{*}}\right) \sqrt{x+1} .
$$

For $\eta(z) \leq 1$, the higher energy photons collide with the same spot size that the electrons would have had so that $\mathcal{L}_{e e}$ is preserved. If we now get the proper intensity for good conversion efficiency without violating the other $z$ constraint we would expect an optimal GLC. Ref. [1] shows how the luminosity spectrum can be controlled. Experiment E144 in Fig. 1 [5] has verified the possibility of $\vec{\gamma}$ beams from the intense laser beams required for efficient conversion. The SLC could usefully verify crossing angles and crab cavities.

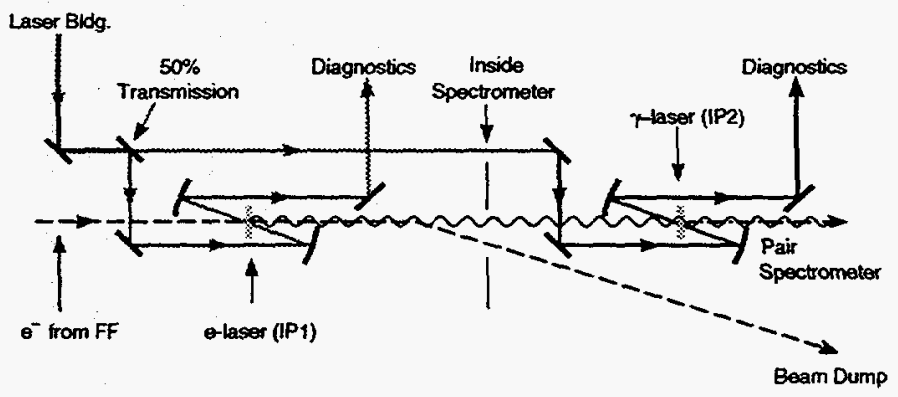

Fig. 1: IPl produces high energy $\gamma s$ and secondary pairs that the 'inside' spectrometer analyzes. Pair spectrometer analyzes the high energy Comptons and pairs from IP2.

\section{B. The Luminosity Budget}

Presently the SLC runs with effectively one bunch/train but having a total current that is 3 times the single bunch value per RF pulse. The simultaneous use of one linac for both beams as well as the scavenger bunch that is used to produce positrons for the next cycle implies an efficiency of 33-67\%. Because BNS damping [6] is used to improve the transverse stability of the bunches it is necessary to correct the phase of the scavenger bunch about midway in the linac which implies an additional inefficiency.

Likewise, just as the scavenger imposes overhead, the use of two charge species increases overhead quite apart from the use of one or two damping rings. Thus, the use of only one species in a train that covers some fraction of the RF fill time will be an improvement. Clearly, operation without the damping rings is also more efficient quite apart from any increase in the current/bunch.

The integration of $R F$ beam monitors with the accelerator implies the possibility of significantly higher single bunch currents [7]. Based on better control of transverse wake fields, more efficient use of charge and much simpler operating conditions we estimate a potential 6-fold increase i.e. $\mathcal{L} \approx 6 \cdot 10^{30}$ without any emittance improvements or increased total charge/pulse.

Although we expect significantly better emittance from $\mathrm{RF}$ guns, we also know that there will be emittance dilution in the arcs e.g.

$$
\Delta \epsilon_{n, x}[\mathrm{~m}] \approx 4 \cdot 10^{-8} E^{6}[\mathrm{GeV}] \mathrm{I}_{5}[1 / \mathrm{m}] \geq 0.8 \cdot 10^{-5}
$$

for point beams with no errors at the $Z$ resonance ( 46.6 $\mathrm{GeV}) . I_{5}$ is the usual synchrotron radiation integral [8].

Assuming an emittance that is a factor of 10 smaller than the SLC $\left(\epsilon_{n} \simeq 3 \cdot 10^{-5} \mathrm{~m}\right)$ for comparable bunch currents, implies it is necessary to run below $40 \mathrm{GeV}$ through the arcs. This implies another acceleration up to final energy even though initial running would be at $10 \mathrm{GeV}$ [1]. Note that a lower energy also allows more total charge. During the final phases it is assumed that either X-Band acceleration at $\sim 100 \mathrm{MeV} / \mathrm{m}$ or laser acceleration at $\sim 1$ $\mathrm{GeV} / \mathrm{m}$ would be available to save space or to accelerate more charge in a bunch of the same length for coalescence. With just the emittance improvement but using the present charge/bunch due to emittance constraints gives about a 20 -fold increase with wake control and simpler operations. 


\section{DISCLAIMER}

Portions of this document may be illegible in electronic image products. Images are produced from the best available original document. 
Increasing the total charge/pulse by a factor of ten is possible [9] with a variety of options. Suppose we allow the increased loading to modify the energy between bunches to take advantage of the longitudinal dispersion in the arcs:

$$
R_{56}=\frac{\delta l_{z}}{\delta p / p}=\int \frac{\eta}{\rho} d s \approx 15 \mathrm{~cm} / \%
$$

where $\delta l$ is the change in bunch separation with a change in bunch energies. While we want to maximize $I_{1}$, we want to minimize $I_{5}$ for the emittance. Thus, while it appears that bunches can be coalesced even for S-Band separations of $10 \mathrm{~cm}$, an X-Band linac with $\lambda \approx 2.6 \mathrm{~cm}$ would provide more flexibility in dealing with the wake field problem in filling near-neighbor buckets. It is possible to cancel some of these effects and even provide acceleration [4] but this appears expensive.

\section{Multi-bunch Trains}

Calculations [9] indicate that a sledded $R F$ pulse could accelerate more than 20 times the present, effective charge/pulse with good characteristics. At lower energies we expect a corresponding increase in pulse rep-rate - all without adding any higher power klystrons [2].

If we accelerate $620 \mathrm{~mA}$ in a $400 \mathrm{~ns}$ train, we increase the total equivalent charge per beam by a factor of 22 assuming $3.5 \times 10^{10}$ per bunch. This provides a maximum possible luminosity gain of 490 . The energy will be reduced by about $10 \mathrm{GeV}$ but this is ideal for preserving the lower emittances from an RF gun. Because we can't coalesce, we have several options to provide a low energy spread e.g. RF amplitude or phase modulation or variation of the beam current with time. In principle, it is possible to get zero energy spread from the acceleration process. This is compatible with lower emittance. Thus, we get another factor of twenty in luminosity from increasing $n_{B}$. Anything near this makes Higgs searches possible.

\section{A Physics Example}

Several options exist while running up to at least $60 \mathrm{GeV}$ for the Higgs but an interesting and practical one is $W^{-}$ production via $\gamma_{R}+e_{L}$. Of particular interest are the zeros in the differential cross sections predicted by the Standard Model when the $W$ goes in the direction of the incoming electron i.e. when the $\gamma$ and $W$ are back-to-back in the center-of-mass. Our interest was the sensitivity of these variations to violations of the Standard Model's predictions at lower energies.

The important process is the 3 gauge boson vertex. The three coupling parameters that conserve $\mathrm{C}$ and $\mathrm{P}$ are determined by the W's charge, magnetic dipole moment $\mu_{W}$ and electric quadrupole moment $\mathrm{Q}_{W}$ :

$$
\mu_{W}=\frac{e}{2 M_{W}}\left(1+\kappa_{\gamma}+\lambda_{\gamma}\right) ; \quad Q_{W}=-\frac{e}{M_{W}^{2}}\left(\kappa_{\gamma}-\lambda_{\gamma}\right) .
$$

Fig. 2 is the SM results for $\sqrt{s}=109$ and $500 \mathrm{GeV}$ where the lower energy is for SLC at $60 \mathrm{GeV}$. While difficult, there is no advantage at higher energies over this low-cost way to study the heavy gauge bosons and their couplings.
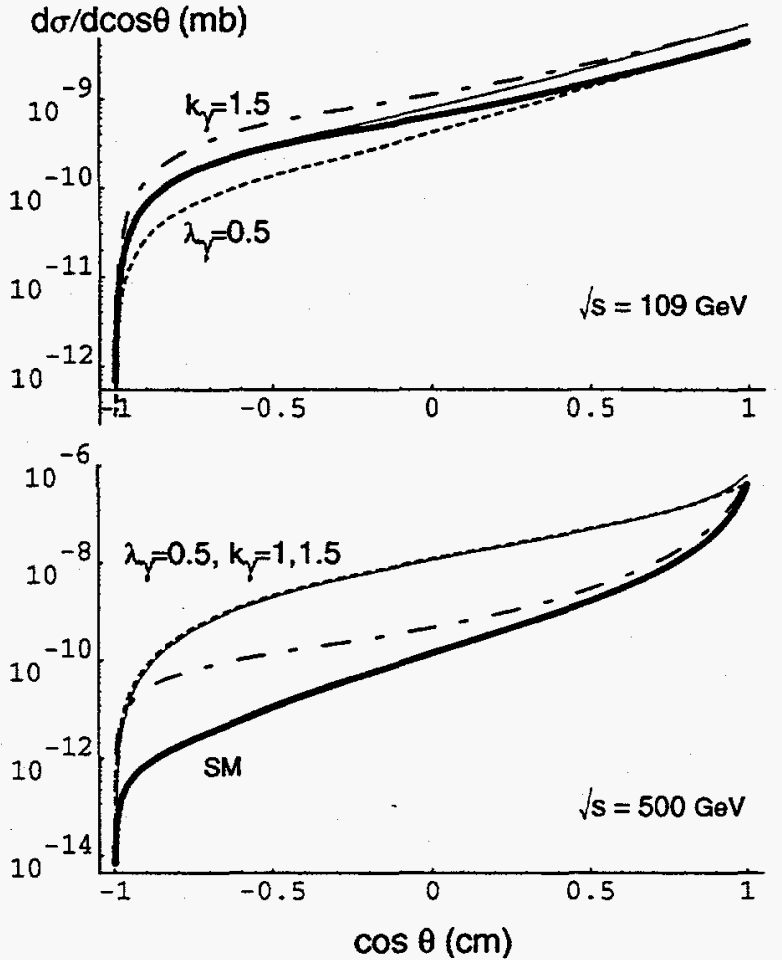

Fig. 2: Differential cross sections for $\gamma_{R}+e_{L} \rightarrow W+$ $\nu$ in the center of mass at $\sqrt{s}=109$ and $500 \mathrm{GeV}$ for the SM prediction of point-like moments $\left(\lambda_{\gamma}=0\right.$ and $\left.k_{\gamma}=1\right)$ and some violations showing sensitivity with energy and angle.

Violations from the lowest order radiative corrections are expected to be of order $\alpha$ and the experiments and machine studies during the various phases are very interesting and have an indeterminant range in energy when we consider future alternatives such as laser acceleration.

\section{References}

[1] J.E. Spencer, Uses of a Prototype NLC/GLC, NIM A355(1995) 184 .

[2] The high-power, S-Band klystron development for DESY implies a peak power increase of nearly 2.5 .

[3] F.-J. Decker, R.K. Jobe, N. Merminga and K.A. Thompson, Controlling Multi-Bunches by a Fast Phase Switching, Lin. Accel. Conf., Sept. 1990.

[4] C.G. Yao and S. Ecklund, Increasing the Energy of SLC by Transient Wake Field, SLAC-PUB-3751, 1985.

[5] K.T. McDonald and A.C. Melissinos, Spokesmen, Proposal for a Study of QED at Critical Field Strength in Intense Laser High-Energy Electron Collisions at SLAC, Proposal E144, Oct. 1991.

[6] V. Balakin, et al., VLEPP: Transverse Beam Dynamics, 12th Int'l.Conf. on High Energy Accel's., 1983.

[7] D. Whittum, Private Communication.

[8] R. Helm, et al., Evaluation of Synchrotron Radiation Integrals, IEEE Trans. Nucl. Sci., 20(1973)900 and T.O. Raubenheimer, et al., PAC Proc., (1993)635.

[9] Z.D. Farkas, Sledded SLAC Beam Loading Compensation, AAS-Note 86, Dec. 1994. I thank David Farkas for discussions on this. 


\section{DISCLAIMER}

This report was prepared as an account of work sponsored by an agency of the United States Government. Neither the United States Government nor any agency thereof, nor any of their employees, makes any warranty, express or implied, or assumes any legal liability or responsibility for the accuracy, completeness, or usefulness of any information, apparatus, product, or process disclosed, or represents that its use would not infringe privately owned rights. Reference herein to any specific commercial product, process, or service by trade name, trademark, manufacturer, or otherwise does not necessarily constitute or imply its endorsement, recommendation, or favoring by the United States Government or any agency thereof. The views and opinions of authors expressed herein do not necessarily state or reflect those of the United States Government or any agency thereof. 